\title{
Effects of electroacupuncture combined with interleukin-10 on chronic sinusitis in mice
}

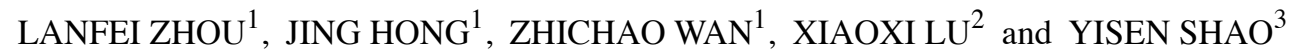 \\ Departments of ${ }^{1}$ Ear, Nose and Throat, ${ }^{2}$ Neurosurgery and ${ }^{3}$ Dentistry, The Affiliated Hospital of \\ Jiangxi University of Traditional Chinese Medicine, Nanchang, Jiangxi 330006, P.R. China
}

Received January 10, 2019; Accepted May 10, 2019

DOI: $10.3892 / \mathrm{mmr} .2019 .10375$

\begin{abstract}
Electroacupuncture (EA) has been documented as a form of therapy for chronic sinusitis (CRS). The present study aimed to assess the effects of EA combined with interleukin-10 (IL-10) overexpression on CRS in mice, and to investigate the associated mechanisms. A mouse model of CRS was established by the administration of ovalbumin (OVA), and overexpression of IL-10 was induced using virus-encoded IL-10. The experimental groups were as follows: i) Control group; ii) OVA group; iii) OVA + EA group; iv) OVA + empty vector group; v) OVA + vector + EA group; vi) OVA + IL-10 group; and vii) OVA + IL-10 + EA group. Pathological changes and nasal mucus were analyzed using hematoxylin and eosin staining. Interferon- $\gamma$ (IFN- $\gamma$ ) and IL-10 were detected via reverse-transcription quantitative PCR and western blot analyses. The pseudostratified epithelium of the mucosa of the nasal sinus was impaired following the induction of CRS. Treatment with EA and/or IL-10 reversed the injury. Combination treatment with EA and IL-10 induced synergistic effects. No infiltration of inflammatory cells was observed in the submucosa following EA and IL-10 treatment. Compared with the control group, the expression of IFN- $\gamma$ and IL-10 in the OVA group was reduced. By contrast, EA or the overexpression of IL-10 inhibited this reduction. Furthermore, the combined application of EA and IL-10 had a significantly more potent inhibitory effect on the reduction of IFN- $\gamma$ expression, but not IL-10. Collectively, EA combined with IL-10 induced specific effects on CRS in mice, likely through the upregulation of IFN- $\gamma$ and IL-10. The current study presented mechanistic implications for the application of EA as an alternative treatment for CRS.
\end{abstract}

Correspondence to: Dr Yisen Shao, Department of Dentistry, The Affiliated Hospital of Jiangxi University of Traditional Chinese Medicine, 445 Bayi Avenue, Nanchang, Jiangxi 330006, P.R. China

E-mail: shaoyisen77@163.com

Key words: chronic sinusitis, interleukin-10, electroacupuncture, interferon- $\gamma$

\section{Introduction}

Chronic sinusitis with nasal polyposis (CRSwNP) is an inflammatory disease of the nasal cavity. Paranasal sinuses develop grape-like structures, leading to nasal obstruction, olfactory disturbance, headache, chronic inflammatory diseases and postnasal drip, which severely affect the quality of life of patients $(1,2)$. CRS is usually caused by repeated bouts of acute inflammation, which lead to pathological changes of the nasal aperture and epithelial ciliary dysfunction (1). The nasal aperture becomes swollen, hypertrophic and polypoid, thereby obstructing drainage of the sinus orifices and leading to CRS (2). The characteristics of CRSwNP include T helper (Th) 2 regulation and eosinophilic inflammation (3).

Interleukin-10 (IL-10) is a potent anti-inflammatory cytokine, which protects the host from excessive tissue damage, and serves a key role in the development and maintenance of immune tolerance and homeostasis (4). The levels of IL-10 are reduced in various chronic inflammatory diseases, including inflammatory bowel disease (5) and spontaneous colitis (6). Imbalance in the levels of IL-10 amplifies the local inflammatory response, which aggravates histopathological changes, and increases the risk of developing autoimmune diseases (7). Consequently, IL-10 is a potential therapeutic target for nasal polyps $(8,9)$.

Electroacupuncture (EA) is a non-drug, adjuvant therapy for certain inflammatory diseases, such as peripheral inflammation (10). It reportedly prevents the nuclear transport of the nuclear factor (NF)- $\mathrm{BB}$ p65 subunit, and thereby inhibits the $N F-\kappa B$ signaling pathway and reduces inflammatory damage (11). Additionally, acupuncture has been used as a therapy for CRS $(12,13)$; however, the mechanisms via which this is achieved have not been fully elucidated. The present study evaluated the therapeutic effects of EA combined with IL-10 overexpression on CRS in mice and investigated the associated mechanisms.

\section{Materials and methods}

Mouse model of CRS. A total of 35 male C57BL/6J mice (3 months of age; body weight $25 \mathrm{~g}$ ) were obtained from Hunan Slake Jingda Laboratory Co., Ltd (Hunan, China) and maintained in specific pathogen-free conditions at a temperature of $23 \pm 2^{\circ} \mathrm{C}$ and a relative humidity of $45-65 \%$ under a controlled 12:12-h light/dark cycle, with access to food and 
water ad libitum. On days 0 and 5, the mice were injected intraperitoneally with $2 \mu \mathrm{g}$ ovalbumin (OVA; cat. no. S7951MSDS; Sigma-Aldrich; Merck KGaA) + 2 mg aluminum hydroxide gel (cat. no. A8222; Sigma-Aldrich; Merck KGaA) to induce CRS as previously described (14). On days 12-19, the animals received $40 \mu \mathrm{l}$ of $3 \%$ OVA nasally. Subsequently, three drops $(\sim 3 \mu \mathrm{l})$ of $3 \%$ OVA were administered per week to induce a long-term inflammatory response over 12 weeks. The control group was treated with a similar volume of saline. All protocols were supervised and approved by the Ethics Committee of Jiangxi University of Chinese Medicine.

Reverse transcription-quantitative PCR (RT-qPCR). Virus-encoded IL-10 (pGreenPuro ${ }^{\mathrm{TM}}$ plasmid packaged in serotype 5 adenovirus) was established by Shanghai GenePharma Co., Ltd. and confirmed in rat cardiac fibroblasts. Adenovirus containing empty pGreenPuro plasmid was used as the control vector.Rat cardiac fibroblasts were purchased from the Cell Bank of Chinese Academy of Sciences, and cultured at $37^{\circ} \mathrm{C}$ with $5 \%$ $\mathrm{CO}_{2}$ in Dulbecco's Modified Eagle's medium (Gibco; Thermo Fisher Scientific, Inc.) supplemented with $10 \%$ fetal calf serum (HyClone; GE Healthcare Life Sciences), $100 \mathrm{U} / \mathrm{ml}$ penicillin and $100 \mathrm{mg} / \mathrm{ml}$ streptomycin. Cells at $70 \%$ confluence were transfected with virus $\left(1 \times 10^{5} \mathrm{IFU} / \mathrm{ml}\right)$ using Lipofectamine ${ }^{\mathrm{TM}}$ 2000 (Invitrogen; Thermo Fisher Scientific, Inc.). At 24 h later, RNA was extracted from cardiac fibroblasts using TRIzol ${ }^{\circledR}$ reagent (Baosheng Science \& Technology Innovation Co., Ltd.) and reverse transcribed into cDNA using an Ultrapure SMART MMLV RT kit (cat. no. 639522, Takara Biotechnology Co., Ltd.) according to the manufacturer's protocol. The cDNA was used as a template for detection by fluorescence qPCR (SYBR Green Master Mix; cat. no. HY-K0501; MedChemExpress LLC) according to the following protocol: 40 cycles of $95^{\circ} \mathrm{C}$ for $10 \mathrm{sec}$ (denaturation), $53^{\circ} \mathrm{C}$ for $30 \mathrm{sec}$ (annealing) and $72^{\circ} \mathrm{C}$ for $30 \mathrm{sec}$ (extension), and a final extension of $72^{\circ} \mathrm{C}$ for $10 \mathrm{~min}$. The relative expression of IL-10 mRNA was calculated with GAPDH as the internal reference using the $2^{-\Delta \Delta \mathrm{Cq}}$ method as previously described (15). The primers are presented in Table I.

Experimental groups. The animals were divided into seven groups ( $\mathrm{n}=5$ in each group) as follows: i) Control group; ii) OVA group; iii) OVA + EA group; iv) OVA + vector group; v) OVA + vector + EA group; vi) OVA + IL-10 group; and vii) OVA + IL-10 + EA group. Mice in the control, OVA and OVA + EA groups were injected with $0.5 \mathrm{ml}$ saline via the tail vein. On day 20 following first injection with OVA, a volume of $0.5 \mathrm{ml}$ of the virus encoding blank vector was injected in the $\mathrm{OVA}+$ vector and OVA + vector + EA groups, whereas $0.5 \mathrm{ml}$ adenovirus encoding IL-10 was injected in the OVA + IL-10 and OVA + IL-10 + EA groups. Following the virus injection, the animals in the OVA + EA, OVA + vector + EA and OVA + IL-10 + EA groups received the first round of electroacupuncture on day 20. Briefly, the animals were anesthetized using isoflurane (1\% in oxygen). EA was conducted in the following corresponding acupoints of mice: i) Zusanli (lateral knee joint, $3.5 \mathrm{~mm}$ below the capitulum fibulae; a 3-mm straight puncture); ii) Feishu (between the ribs below the third thoracic vertebra); iii) Yingxiang (lateral upper end of the nostril, at the hairless junction; a 2-3 mm oblique puncture inward and upward); and iv) Hegu (1-mm straight puncture between the first and second metacarpal bones of the forelimb). Each mouse was stimulated by EA using a Huatuo SDZ-II Electronic Acupuncture Therapy Instrument (Suzhou Medical Supplies Factory Co., Ltd.) at the same acupoint for $30 \mathrm{~min}$ (once a day for 10 days). Following treatment, the animals were anesthetized by isoflurane (1\% in oxygen) via inhalation and decapitated. Nasal tissues were collected on ice using surgical tools under a dissecting microscope (SZ61; Olympus Corporation). Fresh tissues were stored at $-80^{\circ} \mathrm{C}$ or fixed in $4 \%$ paraformaldehyde overnight at $4^{\circ} \mathrm{C}$.

Hematoxylin and eosin staining. Nasal tissues were washed for $1 \mathrm{~h}$ with phosphate-buffered saline (PBS), following which they were fixed with $4 \%$ paraformaldehyde at $4^{\circ} \mathrm{C}$ overnight. The tissues were then dehydrated by 70,80 and $90 \%$ ethanol, and mixed with anhydrous ethanol and xylene for $15 \mathrm{~min}$, xylene I for $15 \mathrm{~min}$ and xylene II for $15 \mathrm{~min}$ (until transparent). Tissues were embedded in paraffin and sliced $(10 \mu \mathrm{m})$. The paraffin slices were dewaxed and hydrated. The sections were stained with hematoxylin for $3 \mathrm{~min}$ at room temperature, differentiated by ethanol hydrochloride for $15 \mathrm{sec}$, washed slightly and stained with eosin for $3 \mathrm{~min}$ at room temperature. The sections were then mounted on slides and observed under a light microscope (magnification, $\mathrm{x} 200$ ).

Immunohistochemistry. Nasal tissues were fixed in $4 \%$ paraformaldehyde overnight at $4^{\circ} \mathrm{C}$. The tissues were subjected to dehydration, embedding and slicing. The paraffin sections $(10 \mu \mathrm{m})$ were then dewaxed and hydrated. Sections were incubated with $3 \%(\mathrm{v} / \mathrm{v})$ hydrogen peroxide for $5 \mathrm{~min}$ at room temperature to block endogenous peroxidase activity. Following blocking in 5\% goat serum (cat. no. G9023; Sigma-Aldrich; Merck $\mathrm{KGaA}$ ) at room temperature for $2 \mathrm{~h}$, the sections were incubated with the following primary antibodies overnight at $4^{\circ} \mathrm{C}$ : Rabbit polyclonal anti-IL-10 (1:300; cat. no. bs-20373R; BIOSS) and rabbit polyclonal anti-interferon- $\gamma$ (IFN- $\gamma ; 1: 300$; cat. no. bs-0480R; BIOSS). The slides were then washed with PBS and incubated with the secondary antibody (horseradish peroxidase-labeled goat anti-rabbit $\operatorname{IgG} ; 1: 10,000$; cat. no. A16104SAMPLE; Thermo Fisher Scientific, Inc.) for $30 \mathrm{~min}$ at room temperature. Sections were subsequently stained with 3,3'-diaminobenzidine chromogen for $3 \mathrm{~min}$ and counterstained with hematoxylin and eosin (3\%, $3 \mathrm{~min})$ at room temperature. Images of four fields for each group were acquired under a light microscope (BX51; Olympus Corporation), and expression density was analyzed by ImageJ software version 1.48 (National Institutes of Health).

Western blotting. Protein was extracted from each group using a protein isolation kit (ReadyPrep; cat. no. 28-9425-44; GE Healthcare Life Sciences). The concentration of the proteins was quantified using a bicinchoninic acid assay. Thereafter, $25 \mu \mathrm{g}$ of protein was separated via $12 \%$ SDS-PAGE. The proteins were then transferred onto polyvinylidene difluoride membranes. The membranes were blocked in $5 \%$ milk for $2 \mathrm{~h}$ at room temperature. The following primary antibodies were incubated with tissues overnight at $4^{\circ} \mathrm{C}$ : Rabbit polyclonal anti-IL-10 (1:300; cat. no. bs-20373R; BIOSS), rabbit polyclonal anti-IFN- $\gamma(1: 300$; cat. no. bs-0480R; BIOSS $)$ and mouse monoclonal anti-GAPDH (1:2,000; cat. no. TA-08; ZSBIO). 
Table I. Primer sequences.

\begin{tabular}{llccc}
\hline \multicolumn{1}{l}{ Gene } & \multicolumn{1}{c}{ Primer $\left(5^{\prime}-3^{\prime}\right)$} & $\begin{array}{c}\text { Primer } \\
\text { length }(\mathrm{bp})\end{array}$ & $\begin{array}{c}\text { Product } \\
\text { length }(\mathrm{bp})\end{array}$ & $\begin{array}{c}\text { Annealing } \\
\text { temperature }\left({ }^{\circ} \mathrm{C}\right)\end{array}$ \\
\hline \multirow{2}{*}{ IL-10 } & Forward, CAACATACTGCTGACAGATTCCT & 23 & 236 & 58.4 \\
& Reverse, GCTCCACTGCCTTGCTTT & 18 & & \\
\multirow{2}{*}{ GAPDH } & Forward, GCAAGTTCAACGGCACAG & 18 & 141 & 58.6 \\
& Reverse, CGCCAGTAGACTCCACGAC & 19 & & \\
\hline
\end{tabular}

Bp, base pairs; IL-10, interleukin-10.

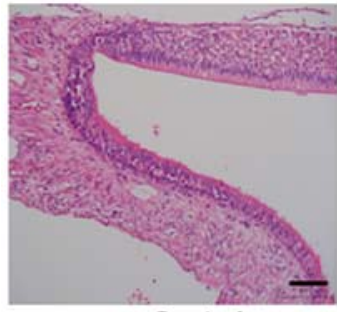

Control

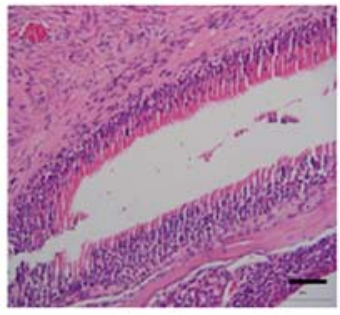

OVA+Vector+EA

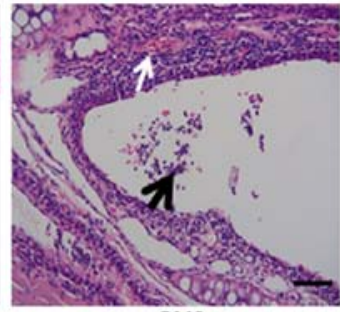

OVA

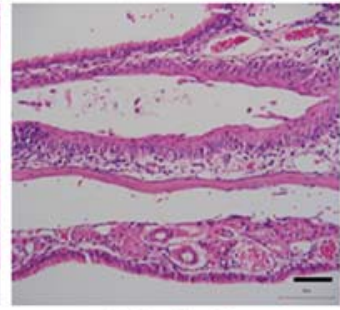

OVA+IL-10

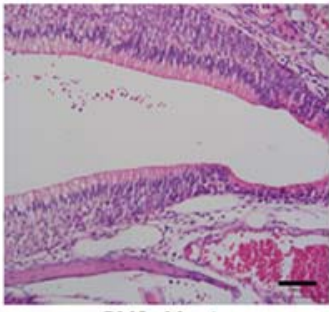

OVA+Vector

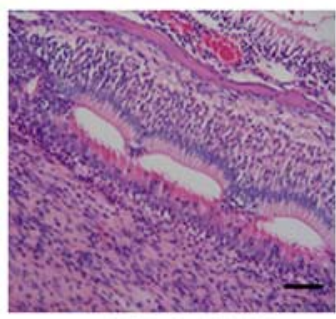

OVA+IL-10+EA

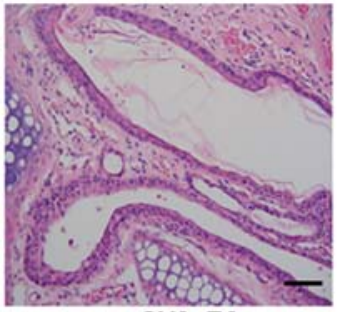

OVA+EA

Figure 1. Treatment with EA and IL-10 ameliorates the injury of the pseudostratified epithelium. Epithelial injury was determined using hematoxylin and eosin staining. The pseudostratified epithelium of the mucosa of the nasal sinuses was impaired in the OVA group and exfoliation was observed (black arrow). Infiltration of inflammatory cells and nuclear condensation were observed in the submucosa (white arrow). Scale bar, $100 \mu \mathrm{m}$. EA, electroacupuncture; IL-10, interleukin-10; OVA, ovalbumin.

The membrane was incubated with peroxidase-conjugated anti-mouse (1:2,000; cat. no. TA130003) or anti-rabbit (1:2,000; cat. no. TA140003; OriGene Technologies, Inc.) secondary antibodies for $2 \mathrm{~h}$ at room temperature. Protein bands were visualized using enhanced chemiluminescence (SuperSignal ${ }^{\mathrm{TM}}$ West Pico Chemiluminescent Substrate; cat. no. 34580; Thermo Fisher Scientific, Inc.). Five repeats were performed, and expression density was analyzed using ImageJ version 1.48.

Statistical analysis. Data are presented as the mean \pm standard error of the mean ( $n=5 /$ group for each experiment). SPSS software (version 17; SPSS, Inc.) was used to analyze the data. One-way analysis of variance was applied to the data, followed by the Newman-Keuls post-hoc test. $\mathrm{P}<0.05$ was considered to indicate a statistically significant difference.

\section{Results}

Morphological changes in the mucosa of the nasal sinuses following establishment of the CRS model. As presented in Fig. 1, the pseudostratified epithelium of the mucosa of the nasal sinuses in the control group exhibited a neatly arranged, complete structure. No infiltration of inflammatory cells was observed

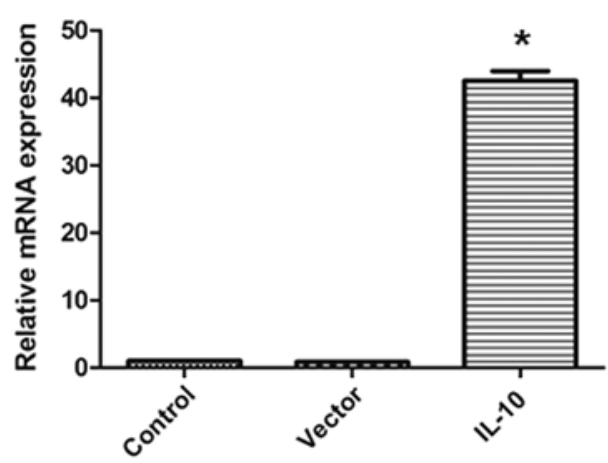

Figure 2. Confirmation of IL-10 expression in fibroblasts. Expression of IL-10 was significantly increased in the virus-encoded IL-10 group compared with the control group. ${ }^{*} \mathrm{P}<0.05$ vs. control. IL-10, interleukin-10.

in the submucosa. By contrast, the OVA group showed severe inflammation, a disordered arrangement of mucosal epithelium, necrosis and exfoliation. These results indicated that injury of pseudostratified epithelium was induced in the CRS model.

Virus-encoded IL-10 promotes IL-10 expression. As presented in Fig. 2, the expression of IL-10 in cardiac fibroblasts 

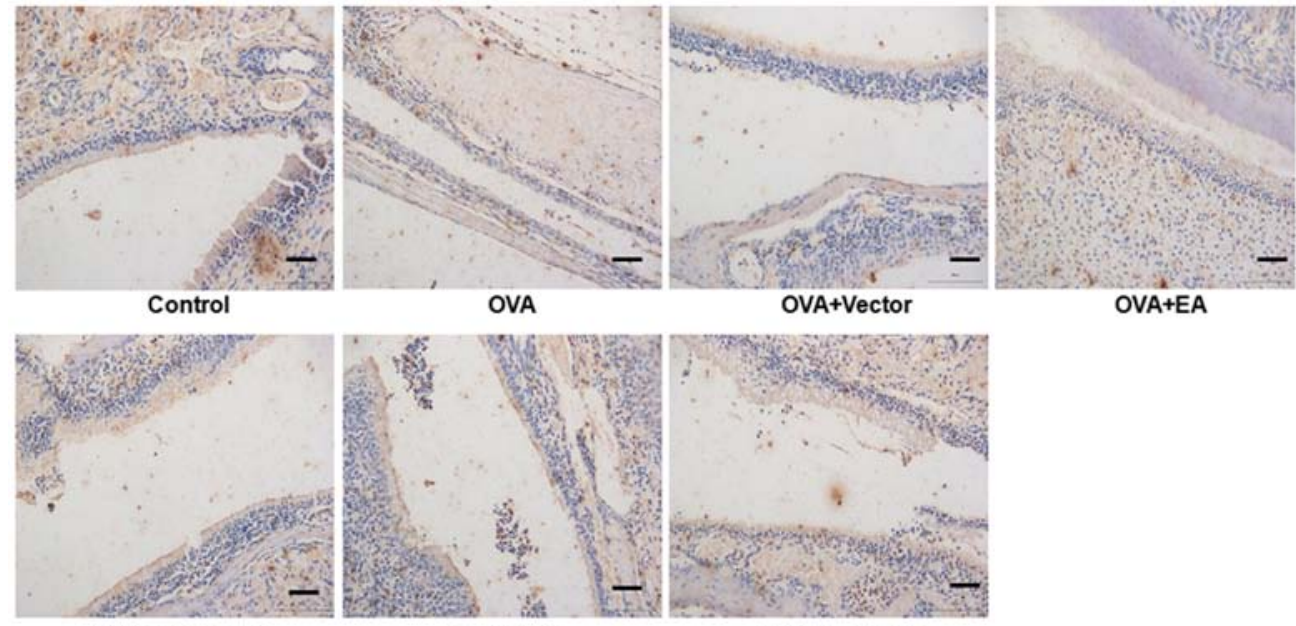

OVA+EA

OVA+Vector+EA

OVA+IL-10

OVA+IL-10+EA

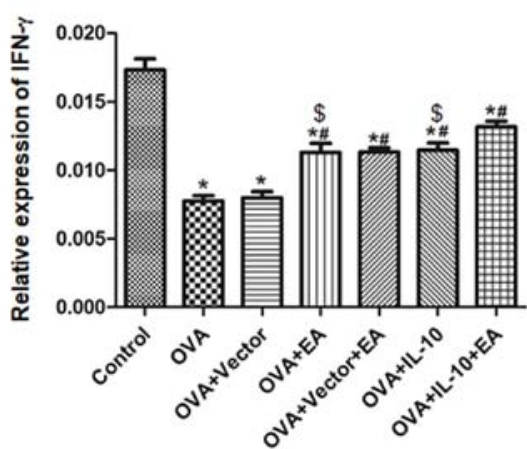

Figure 3. Treatment with EA and IL-10 rescues IFN- $\gamma$ expression. Immunohistochemistry revealed that IFN- $\gamma$ expression was decreased in the OVA group, but promoted by EA and virus-encoded IL-10. Upper panel, representative images of each group. Scale bar, $100 \mu \mathrm{m}$. Lower panel, IFN- $\gamma$ protein level quantification. ${ }^{*} \mathrm{P}<0.05$ vs. control; ${ }^{\#} \mathrm{P}<0.05$ vs. OVA; ${ }^{\$} \mathrm{P}<0.05$ vs. OVA+IL-10+EA. EA, electroacupuncture; IL-10, interleukin-10; IFN- $\gamma$, interferon- $\gamma$; OVA, ovalbumin.

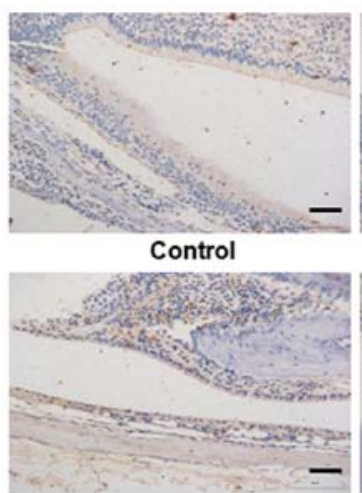

OVA+Vector+EA

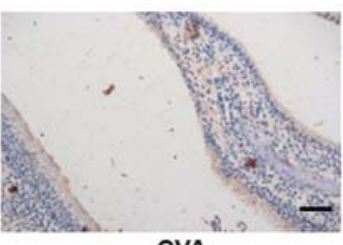

OVA

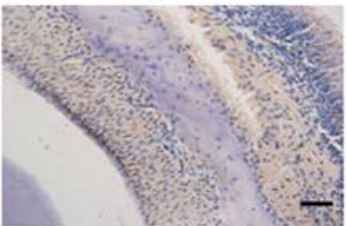

OVA+IL-10

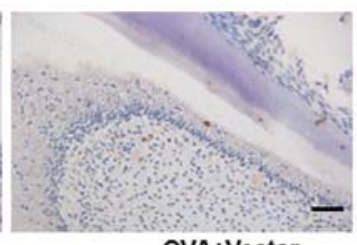

OVA+Vector

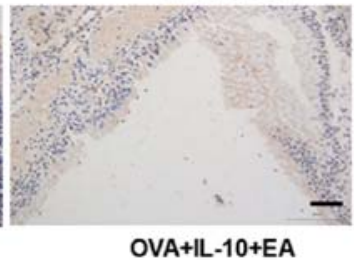

OVA+IL-10+EA

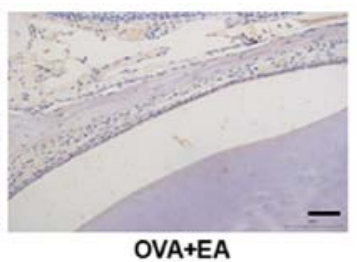

OVA+EA

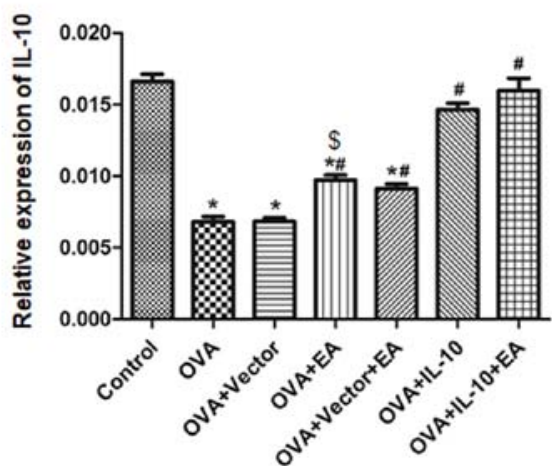

Figure 4. Treatment with EA and IL-10 rescues IL-10 expression. Immunohistochemistry showed that IL-10 expression was decreased in the model group, but promoted by EA and virus-encoded IL-10. Upper panel, representative images of each group. Lower panel, IL-10 protein level quantification. ${ }^{*} \mathrm{P}<0.05$ vs. control; ${ }^{\#} \mathrm{P}<0.05$ vs. OVA; ${ }^{~} \mathrm{P}<0.05$ vs. OVA+IL-10+EA. Scale bar, $100 \mu \mathrm{m}$. EA, electroacupuncture; IL-10, interleukin-10; OVA, ovalbumin. 
A
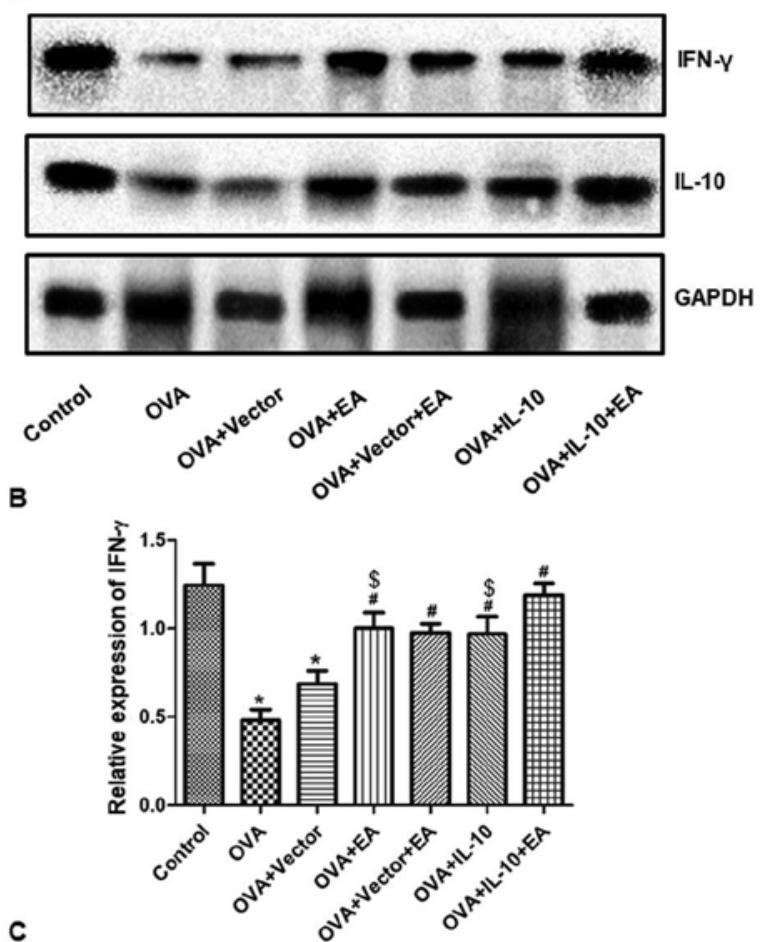

C

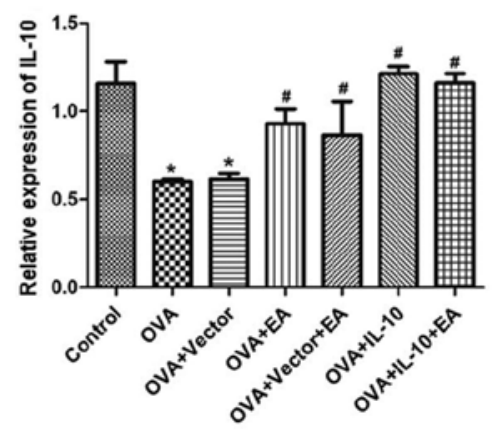

Figure 5. Treatment with EA and IL-10 increases IL-10 and IFN- $\gamma$ expression. (A) Representative blots of IFN- $\gamma$ and IL-10. (B) Western blotting showed that IFN- $\gamma$ expression was decreased in the model group, but promoted by EA and virus-encoded IL-10. (C) Western blotting showed that IL-10 expression was decreased in the model group, but promoted by $\mathrm{EA}$ and virus-encoded IL-10. ${ }^{*} \mathrm{P}<0.05$ vs. control; ${ }^{*} \mathrm{P}<0.05$ vs. OVA ${ }^{\$} \mathrm{P}<0.05$ vs. OVA+IL-10+EA. EA, electroacupuncture; IL-10, interleukin-10; IFN- $\gamma$, interferon- $\gamma$; OVA, ovalbumin.

transduced with virus-encoded IL-10 was significantly increased compared with in the control group $(\mathrm{P}<0.05)$, whereas the empty vector virus did not affect IL-10 expression. Therefore, the virus-encoded IL-10 was used in the subsequent experiments.

Treatment with EA and IL-10 ameliorates injury of the pseudostratified epithelium. The OVA group and OVA + vector group exhibited severe inflammation, disordered arrangement of the mucosal epithelium, necrosis and exfoliation. Treatment with EA (OVA + EA group) or IL-10 (OVA + IL-10 group) resulted in attenuation of the injury induced by the CRS model (Fig. 1). Notably, combination therapy with EA and IL-10 was more effective than treatment with EA or IL-10 alone. In the OVA + IL-10 + EA group, cells were neatly arranged and few inflammatory cells were observed (Fig. 1).
Treatment with EA and IL-10 increases IFN- $\gamma$ and IL-10 expression. The results of immunohistochemistry are presented in Fig. 3. Compared with the control group, the expression of IFN- $\gamma$ in the OVA group was significantly decreased $(\mathrm{P}<0.05)$. Compared with the OVA group, the expression of IFN- $\gamma$ was promoted by EA (OVA + EA group) and virus-encoded IL-10 (OVA + IL-10 group; $\mathrm{P}<0.05)$, but not by the virus-encoding vector (OVA + vector group). The combined effect of EA and IL-10 (OVA + IL-10 + EA group) was significantly increased compared with EA or IL-10 alone $(\mathrm{P}<0.05)$.

The results of IL-10 expression are shown in Fig. 4. Compared with the control group, the expression of IL-10 in the OVA group was significantly decreased $(\mathrm{P}<0.05)$. Compared with the OVA group, the expression of IL-10 was promoted by EA (OVA + EA group) and virus-encoded IL-10 (OVA + IL-10 group; $\mathrm{P}<0.05$ ), but not by the virus-encoding vector (OVA + vector group). Combined treatment with EA did not further promote IL-10 expression compared with IL-10 overexpression alone $(\mathrm{P}>0.05)$.

The expression of IFN- $\gamma$ and IL-10 was also quantified by western blotting. Consistently, OVA treatment reduced IFN- $\gamma$ and IL-10 expression, whereas EA or IL-10 overexpression promoted the expression of IL-10 and IFN- $\gamma$ in OVA-treated mice. The combination of EA and overexpression of IL-10 further promoted IFN- $\gamma$, but not IL-10 levels, compared with the individual treatments (Fig. 5).

\section{Discussion}

The current study provided novel data demonstrating the protective effects of EA and IL-10 on CRS in mice. Based on the morphological changes observed, EA and virus-encoded IL-10 ameliorated the injured pseudostratified epithelium. Notably, the combined application of EA and IL-10 expression induced additive effects, likely via the upregulation of IFN- $\gamma$.

An animal model of sinusitis is important for the basic and clinical research of this type of disease. The stability and repeatability of the model also guarantee progress of the research. From the early 20th century, animal models have been used in sinusitis research, and OVA has been used as an agent to induce CRS (16). In the current study, OVA was used to induce CRS, whereas aluminum hydroxide gel was used to protect against gastric injury induced by OVA, and morphological changes of the mucosa of the nasal sinus were observed (14). The pseudostratified epithelium of the mucosa of the nasal sinus was impaired in the model group, and was characterized by severe inflammation, disordered arrangement of the mucosal epithelium, necrosis and exfoliation, all of which are symptoms of sinusitis (14). Furthermore, EA treatment ameliorated the abnormal changes of the mucosa. The results obtained in the current study suggested that the mouse model of sinusitis was successfully induced, and that EA exerted protective effects in this model.

As a major anti-inflammatory cytokine, IL-10 serves important roles in tumors, infections, organ transplantation, and the hematopoietic and cardiovascular systems $(17,18)$. Furthermore, IL-10 is closely associated with the blood, digestion and diseases of the cardiovascular system (19). Impaired IL-10 production, in response to Staphylococcus aureus 
enterotoxin B in nasal polyps, likely exacerbates the pathophysiological mechanisms of eosinophilic chronic rhinosinusitis, including eosinophilia and obstruction of the lower airway (20). In the present study, IL-10 levels were reduced in the OVA group compared with the control group. By contrast, EA treatment promoted IL-10 expression. These data suggested that EA likely exerted protective effects in CRS by increasing IL-10 levels. IL-10 upregulation has a protective effect in CRSwNP $(8,9)$. In allergic fungal rhinosinusitis, IL-10 levels were reportedly reduced (21), indicating that IL-10 may be a potential therapeutic target for the treatment of CRSwNP caused by various pathologic factors (22). In the present study, EA alone improved IL-10 expression in the CRS model, but in combination with IL-10 overexpression, did not further promote IL-10 expression. IL-10 acts by blocking the metabolism of macrophages as part of this inflammatory response (CD4+ T-cell responses) (23). Specifically, IL-10 is able to inhibit lipopolysaccharide-induced glucose uptake and glycolysis, and promote oxidative phosphorylation (24). In addition, a reduction in IL-10 levels damaged mitochondria by promoting mitochondrial autophagy, and subsequently eliciting inflammation (25).

The IFN- $\gamma$ cytokine has antiviral, immunomodulatory and antitumor properties $(26,27)$. In addition to inducing the differentiation and maturation of Th1 cells and increasing IFN- $\gamma$ levels in the serum, EA inhibits the differentiation and maturation of Th 2 cells and the production of cytokines (28). Thus, EA inhibits the transformation of Thl cells to Th 2 cells, and thereby corrects any imbalance in the number of Thl/2 cells $(28,29)$ and enhances the cellular immune response. In the present study, IL-10 overexpression attenuated the reduction in IFN- $\gamma$ levels observed in the OVA model. Furthermore, EA alone promoted IFN- $\gamma$ expression. These findings suggested that IL-10 may act upstream of IFN- $\gamma$ in immunotherapy. Indeed, IL-10 controlled IFN- $\gamma$ and the production of tumor necrosis factor during experimental endotoxemia (30). However, IFN- $\gamma$ could also reprogram IL-10 activity in macrophages (31). Nevertheless, the cooperative actions of IFN- $\gamma$ and IL-10 in the regulation of cell functions cannot be ignored (32). In the current study, EA did not further promote IL-10 levels but increased IFN- $\gamma$ when applied in combination with IL-10 overexpression. These findings suggested that EA may function via various mechanisms to promote IFN- $\gamma$ expression.

Treatment with EA may promote the secretion of adrenocortical hormones, thereby enhancing the function of the pituitary-adrenal cortex and sympathetic-adrenal systems to improve resistance to certain diseases, such as myocardial ischemia injury (33). In addition, EA therapy enhanced immunity by improving the phagocytic ability of the mononuclear macrophage system (34) and function of T helper cells (35) in rats. Moreover, EA increased microvascular perfusion, improved blood circulation, supported the lymphatic and nervous systems and regulated cellular and humoral immunity in humans (36). Thus, the exact mechanisms of EA in the treatment of CRSwNP require further investigation.

The present study possessed to certain limitations. EA promoted IL-10 expression and IL-10 overexpression ameliorated the pathological changes of CRSwNP; however, the underlying mechanisms were not elucidated. The manner via which IL-10 regulates IFN- $\gamma$ requires clarification. An increased number of quantified parameters to evaluate pathological changes should be detected to confirm the effectiveness and uniformity of the treatment administered by nasal drops. Finally, a transgenic model, such as IL-10 knockout mice, may be more effective in determining the underlying mechanisms.

In conclusion, EA and IL-10 effectively inhibit inflammation and increase the expression of IFN- $\gamma$, and thereby exert various specific effects in the treatment of CRS in mice. These data may provide novel insight for the future treatment of CRS.

\section{Acknowledgements}

Not applicable.

\section{Funding}

The current study was supported by grants from the Jiangxi Education Department, the Jiangxi Provincial Health Planning Commission (grant no. 2016A102) and the State Administration of Chinese Medicine (grant no. 201507006).

\section{Availability of data and materials}

The datasets used and/or analyzed during the current study are available from the corresponding author on reasonable request.

\section{Authors' contributions}

$\mathrm{LZ}$ and YS conceived and designed the experiments. $\mathrm{JH}$, ZW and XL performed the experiments and analyzed the data. LZ and YS wrote the manuscript. All authors read and approved the manuscript and agree to be accountable for all aspects of the research in ensuring that the accuracy or integrity of any part of the work are appropriately investigated and resolved.

\section{Ethics approval and consent to participate}

The experimental protocols were approved by the Ethics Committee of Jiangxi University of Chinese Medicine.

\section{Patient consent for publication}

Not applicable.

\section{Competing interests}

The authors declare that they have no competing interests.

\section{References}

1. Li XZ,Zhao SC, Cai XL, Wang YF, Chen J, Ma XF and Zhang H: Differences in expression of YKL-40 and TLR4 in nasal sinus mucosa of chronic sinusitis patients with and without nasal polyps. J Biol Regul Homeost Agents 32: 537-543, 2018.

2. Fokkens WJ, Lund VJ, Mullol J, Bachert C, Alobid I, Baroody F, Cohen N, Cervin A, Douglas R, Gevaert P, et al: European position paper on rhinosinusitis and nasal polyps 2012. Rhinol Suppl 23: 3 p preceding table of contents, 1-298, 2012.

3. Bachert C, Zhang L and Gevaert P: Current and future treatment options for adult chronic rhinosinusitis: Focus on nasal polyposis. J Allergy Clin Immunol 136: 1431-1440, 2015. 
4. Schmetterer KG and Pickl WF: The IL-10/STAT3 axis: Contributions to immune tolerance by thymus and peripherally derived regulatory T-cells. Eur J Immunol 47: 1256-1265, 2017.

5. Shah N, Kammermeier J, Elawad $M$ and Glocker EO Interleukin-10 and interleukin-10-receptor defects in inflammatory bowel disease. Curr Allergy Asthma Rep 12: 373-379, 2012.

6. Lennon EM, Borst LB, Edwards LL and Moeser AJ: Mast cells exert anti-inflammatory effects in an IL10 ${ }^{-/-}$model of spontaneous colitis. Mediators Inflamm 2018: 7817360, 2018.

7. Iyer SS and Cheng G: Role of interleukin 10 transcriptional regulation in inflammation and autoimmune disease. Crit Rev Immunol 32: 23-63, 2012

8. Xu J, Han R, Kim DW, Mo JH, Jin Y, Rha KS and Kim YM: Role of interleukin-10 on nasal polypogenesis in patients with chronic rhinosinusitis with nasal polyps. PLoS One 11: e0161013, 2016

9. Wang C, Lou H, Wang X, Wang Y, Fan E, Li Y, Wang H, Bachert $C$ and Zhang L: Effect of budesonide transnasal nebulization in patients with eosinophilic chronic rhinosinusitis with nasal polyps. J Allergy Clin Immunol 135: 922-929.e6, 2015

10. Kim HW, Kang SY, Yoon SY, Roh DH, Kwon YB, Han HJ, Lee HJ, Beitz AJ and Lee JH: Low-frequency electroacupuncture suppresses zymosan-induced peripheral inflammation via activation of sympathetic post-ganglionic neurons. Brain Res 1148 69-75, 2007.

11. Chen X, Tang C, Xie H, Tang N, Gao J and Liu R: Effect of electro-acupuncture on hepatic Toll-like receptor 4 and nuclear factor $\kappa \mathrm{B}$ expressions in rats with non-alcoholic fatty liver disease. Nan Fang Yi Ke Da Xue Xue Bao 34: 1584-1588, 2014 (In Chinese)

12. Rössberg E, Larsson PG, Birkeflet O, Söholt LE and Stavem K: Comparison of traditional Chinese acupuncture, minimal acupuncture at non-acupoints and conventional treatment for chronic sinusitis. Complement Ther Med 13: 4-10, 2005.

13. Kim JI, Choi JY, Lee MS, Kim TH, Kim AR, Jung SY, Shin MS and $\mathrm{Kim} \mathrm{KH:} \mathrm{Acupuncture} \mathrm{for} \mathrm{improving} \mathrm{chronic} \mathrm{rhinosinus-}$ itis complicated with persistent allergic rhinitis. A prospective observational study. Forsch Komplementmed 17: 333-335, 2010.

14. Kim DY, Lee SH, Carter RG, Kato A, Schleimer RP and Cho SH: A recently established murine model of nasal polyps demonstrates activation of B cells, as occurs in human nasal polyps. Am J Respir Cell Mol Biol 55: 170-175, 2016.

15. Livak KJ and Schmittgen TD: Analysis of relative gene expression data using real-time quantitative PCR and the 2(-Delta Delta C(T)) method. Methods 25: 402-408, 2001

16. Mendiola M, Tharakan A, Chen M, Asempa T, Lane AP and Ramanathan M Jr: Characterization of a novel high-dose ovalbumin-induced murine model of allergic sinonasal inflammation. Int Forum Allergy Rhinol 6: 964-972, 2016.

17. Ip WKE, Hoshi N, Shouval DS, Snapper S and Medzhitov R: Anti-inflammatory effect of IL-10 mediated by metabolic reprogramming of macrophages. Science 356: 513-519, 2017.

18. Rossi O, van Berkel LA, Chain F, Tanweer Khan M, Taverne N Sokol H, Duncan SH, Flint HJ, Harmsen HJ, Langella P, et al: Faecalibacterium prausnitzii A2-165 has a high capacity to induce IL-10 in human and murine dendritic cells and modulates T cell responses. Sci Rep 6: 18507, 2016.

19. Jung M, Ma Y, Iyer RP, DeLeon-Pennell KY, Yabluchanskiy A, Garrett MR and Lindsey ML: IL-10 improves cardiac remodeling after myocardial infarction by stimulating M2 macrophage polarization and fibroblast activation. Basic Res Cardiol 112: 33, 2017.

20. Haruna T, Kariya S, Fujiwara T, Higaki T, Makihara S, Kanai K, Fujiwara R, Iwasaki S, Noguchi Y, Nishizaki K and Okano M: Association between impaired IL-10 production following exposure to Staphylococcus aureus enterotoxin B and disease severity in eosinophilic chronic rhinosinusitis. Allergol Int 67: 392-398, 2018.

21. Rai G, Das S, Ansari MA, Singh PK, Gupta N, Sharma S, Akhter N, Ramachandran VG, Haque S and Dar SA: Phenotypic and functional profile of Th17 and Treg cells in allergic fungal sinusitis. Int Immunopharmacol 57: 55-61, 2018.
22. Wang YX, Gu ZW, Cao ZW and Hao LY: Nonylphenol can aggravate allergic rhinitis in a murine model by regulating important Th cell subtypes and their associated cytokines. Int Immunopharmacol 70: 260-267, 2019.

23. Nova-Lamperti E, Fanelli G, Becker PD, Chana P, Elgueta R, Dodd PC, Lord GM, Lombardi G and Hernandez-Fuentes MP: IL-10-produced by human transitional B-cells down-regulates CD86 expression on B-cells leading to inhibition of $\mathrm{CD}^{+} \mathrm{T}$-cell responses. Sci Rep 6: 20044, 2016.

24. Wang Y, Yi J, Chen X, Zhang Y, Xu M and Yang Z: The regulation of cancer cell migration by lung cancer cell-derived exosomes through TGF- $\beta$ and IL-10. Oncol Lett 11: 1527-1530, 2016.

25. Hayashi M, Yanaba K, Umezawa Y, Yoshihara Y, Kikuchi S, Ishiuji Y, Saeki H and Nakagawa H: IL-10-producing regulatory B cells are decreased in patients with psoriasis. J Dermatol Sci 81: 93-100, 2016

26. Kawamoto S, Oritani K, Asakura E, Ishikawa J, Koyama M, Miyano K, Iwamoto M, Yasuda S, Nakakubo H, Hirayama F, et al: A new interferon, limitin, displays equivalent immunomodulatory and antitumor activities without myelosuppressive properties as compared with interferon-alpha. Exp Hematol 32: 797-805, 2004.

27. Sakai S, Kauffman KD, Sallin MA, Sharpe AH, Young HA, Ganusov VV and Barber DL: CD4 T cell-derived IFN- $\gamma$ plays a minimal role in control of pulmonary mycobacterium tuberculosis infection and must be actively repressed by PD-1 to prevent lethal disease. PLoS Pathog 12: e1005667, 2016.

28. Yuliatun L, Amalia SH, Rahma AA and Yaumi LA: Electro-acupuncture therapy increases serum interferon- $\gamma$ levels in rats with 7,12 Dimethylbenz $(\alpha)$ anthracene (DMBA)-induced breast tumors. Asian Pac J Cancer Prev 18: 1323-1328, 2017.

29. Carneiro ER, Xavier RA, De Castro MA, Do Nascimento CM and Silveira VL: Electroacupuncture promotes a decrease in inflammatory response associated with Th1/Th2 cytokines, nitric oxide and leukotriene B4 modulation in experimental asthma. Cytokine 50: 335-340, 2010.

30. Marchant A, Bruyns C, Vandenabeele P, Ducarme M, Gérard C, Delvaux A, De Groote D, Abramowicz D, Velu T and Goldman M: Interleukin-10 controls interferon-gamma and tumor necrosis factor production during experimental endotoxemia. Eur J Immunol 24: 1167-1171, 1994.

31. Herrero C, Hu X, Li WP, Samuels S, Sharif MN, Kotenko S and Ivashkiv LB: Reprogramming of IL-10 activity and signaling by IFN-gamma. J Immunol 171: 5034-5041, 2003.

32. Yanagawa $\mathrm{Y}$, Iwabuchi $\mathrm{K}$ and Onoe $\mathrm{K}$ : Co-operative action of interleukin-10 and interferon-gamma to regulate dendritic cell functions. Immunology 127: 345-353, 2009.

33. Cui S,Zhou Y,Wu S,Cao J,Zhu G and Zhou M:Electroacupuncture improved the function of myocardial ischemia involved in the hippocampus-paraventricular nucleus-sympathetic nerve pathway. Evid Based Complement Alternat Med 2018: 2870676, 2018.

34. Zeng Q, He H, Wang XB, Zhou YQ, Lin HX, Tan ZP, He SF and Huang GZ: Electroacupuncture preconditioning improves myocardial infarction injury via enhancing AMPK-dependent autophagy in rats. Biomed Res Int 2018: 1238175, 2018.

35. Zhu J, Chen XY, Li LB, Yu XT, Zhou Y, Yang WJ, Liu Z, Zhao N, Fu C, Zhang SH and Chen YF: Electroacupuncture attenuates collagen-induced arthritis in rats through vasoactive intestinal peptide signalling-dependent re-establishment of the regulatory T cell/T-helper 17 cell balance. Acupunct Med 33: 305-311, 2015.

36. Tan CF, Yan J, Wang C, Chang XR, Xie WJ, Yang JJ, Liu M, Lin HB and He XC: Effects of electroacupuncture and moxibustion pretreatment on expressions of HSP 27, HSP 70, HSP 90 at different time-points in rabbits with myocardial ischemia-reperfusion injury. Zhen Ci Yan Jiu 42: 31-38, 2017 (In Chinese). 